\title{
Calculation Principle and Test Applications for Digital Speckle Correlation Technology
}

\author{
Yongsheng Liu*, Zhang-lin CHEN, Wang Liu and Xinyu Dong
}

School of Civil Engineering and Architecture, East China Jiaotong University, Nanchang, Jiangxi, 330013, China

\begin{abstract}
With SSD algorithm and calculus process in detail computing, the basic calculation principles and the solution process from digital image to displacement of digital speckle correlation technology were described. Then the digital speckle correlation technology were used to the tensile test of CFRP plate embedded reinforcement beams, obtained the displacement cloud image and destruction rules of the specimens. The results show that the calculation results analyzed by the digital image correlation technology are in good agreement with the experimental phenomena. And the technology has the characteristic of full field measurement, simple operation, strong adaptability and high precision, so it has the widespread application prospect, especially suitable for application in the mechanical properties of materials under complex conditions, a large section of the destruction process monitoring and other fields.
\end{abstract}

Keywords: Beam strengthened with CFRP plate, correlation function, digital speckle correlation technology, SSD algorithm.

\section{INTRODUCTION}

According to the different ways of measuring, testing techniques can be divided into contact and non-contact. Mechanical instrumentation method, displacement sensor method and the resistance strain gauge method are the primary method of contact measurement. Currently, Because of its high accuracy and simple operation, the resistance strain gauge method was most widely used in engineering. However, the resistance strain gauge method relies on the strain gauge to measure, the method can only measure partial small strain, and the flexible specimens are difficult to measure; Also, under some special circumstances, such as high temperature or surface corrosion, it is difficult to paste the strain gauge, the resistance strain gauge method are difficult to apply. Optical measurement is the most important non-contact measurement method. The data collection of traditional optical method is the use of film or dry plate recording light intensity distribution to get the physical displacement or deformation of the surface. $\mathrm{NOH}$ MYOUNG GYU [1] invented the apparatus of non-contact mass measurement in 2014. Patent 2012120602(A) [2] ,tiled "Device for the remote non-contact monitoring of the vital signs of a living being" provides the monitoring method of the vital signs. They all promoted the application of noncontact measurement technology. However, this method with time-consuming and difficult to accurately control, it is not conducive to the subsequent image processing and computer use. Digital speckle technique is a rapid development of optical measurement technology, with high-precision, data collection simple and low measuring environmental requirements, its advantages have been widely recognized.

*Address correspondence to this author at the School of Civil Engineering and Architecture, East China Jiaotong University, Nanchang, Jiangxi, 330013, China; Tel: 85-971-87046707; Fax: 85-971-87046720;

E-mail: yshliu07@126.com
In recent years, many scholars began to study digital speckles technology and its applications are constantly expanding. Patent CN102967261 (A) [3], titled "Laser displacement measuring method based on digital speckle correlation method" provided the application of speckle correlation to the measuring method. This all promote the development of the technology. But the technology involves mechanics and optics, the theory is complicated. This article briefly describes the basic principles of digital speckle technology and detailed studies its basic algorithm. Finally, the technology was used to CFRP plate embedded in reinforced concrete beams deformation testing. The test results are consistent with the experimental results which illustrate the rationality of the theoretical analysis.

\section{SUMMARY OF DIGITAL SPECKLE TECHNOLOGY}

Digital image correlation was originally proposed by the research team of Professor Ichiro Yamaguchi, Japan and the University of South Carolina in the 1980s [4, 5]. Peter and Ransom [6] of University of South Carolina, used digital imaging technology measuring component of surface displacement, using television camera for recording the laser speckle pattern before and after loading, after analog-digital conversion, the image is stored in the computer to obtain digital gray field. Comparing the two images, by calculate the extreme of correlation coefficient obtained surface deformation of the object. And patent 103686256(A) provides a method and system for obtaining information by television camera [7]. In study of small deformation of objects, Ichiro Yamaguchi [4, 5] proposed a non-contact laser speckle strain gauge. Patent CN101850426 (A), titled "Manufacturing method of high-temperature speckle" [8] provided the method manufacturing speckle on the surface of high-temperature material. Peak of the correlation function 


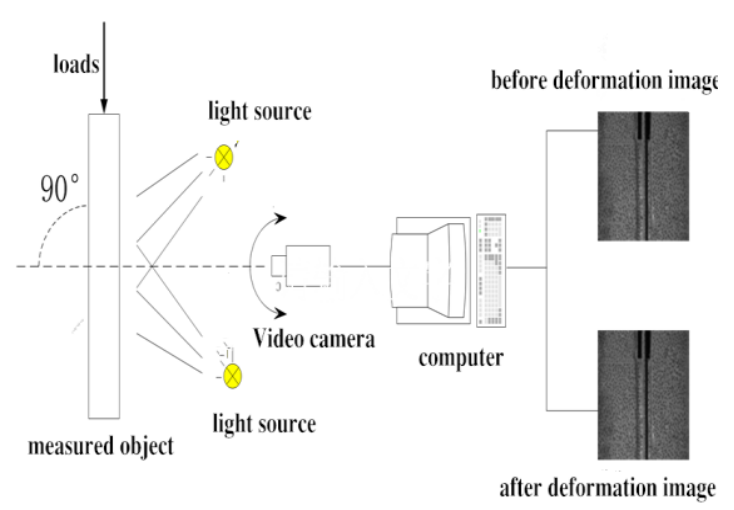

Fig. (1). Digital speckle correlation measurement system.

obtained by the photodiode array, and the displacement of the object can be deduced from it.

Research started in China for digital image correlation technique in the late 1980s, Jianxin Gao etc. [9, 10] carried out the research of the digital speckle correlation technique firstly, then many domestic scholars have done a lot of research on this technology. They all made more great development, especially with the development of computer technology, the technology in computing speed, accuracy have made some breakthroughs, be fully applied in various fields.

The basic principles of digital speckle correlation technology is match on the surface digitized image speckle geometric points in different states, tracking motion points to obtain deformation information on object's surface deformation. In the experiment, the two gray-scale image of the object was collected before and after deformation. To establish contact through the correlation function and gray information, correspondence between the two images by the sub-region, the amount of deformation and strain can be obtained.

\section{SSD ALGORITHM}

\subsection{Calculation Principle}

Take two speckle fields before and after deformation of the object and the fields are divided into $\mathrm{M}$ rows and $\mathrm{N}$ columns, forming a matrix form, each of the small areas called pixels, and with a value representative. This value represents the area corresponding to the gradation value of the analog image in this position. In this case, each of the small areas is an exact gray value. The values of all these positions are filled matrix; the sub-matrixre presents the digital images of this graph [11-12]. Select the appropriate size of sample patterns, as the speckle pattern before deformation, the purpose of this calculation is to find the deformed target sub-region. Show as Fig. (2). Patent 20080013853A1 provides a method for processing a digital gray value image [13].

During calculating, the choice of the appropriate correlation function algorithm is very important for find the target sub-region. Current the correlation functions risen up by scholar at home and abroad are nearly ten. Sum of squared differences (SSD) [14] is a basic function algorithm. The formula can be expressed as:

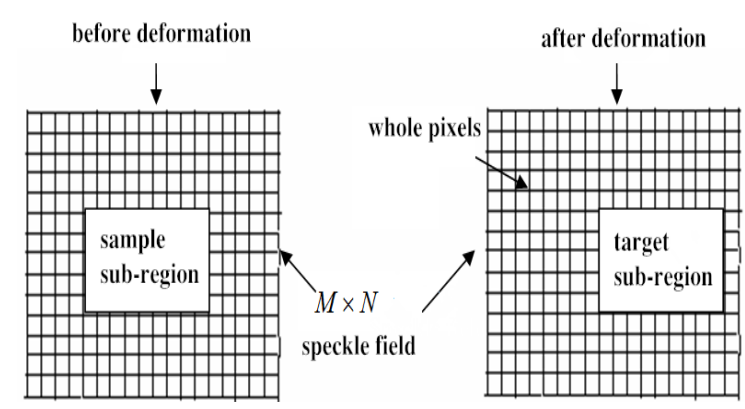

Fig.(2). Geometric variations of the sub-region before and after deformation.

$C_{S S D}=\sum_{i=-M}^{M} \sum_{j=-N}^{N}\left[F_{1}\left(x_{i} y_{j}\right)-F_{2}\left(x_{i} y_{j}\right)\right]^{2}$

Where, $F_{1}\left(x_{i} y_{j}\right)$ is the initial image gray value function, $F_{2}\left(x_{i} y_{j}\right)$ is the image gray value function after deformation. Comparison of the two images, it is to solve the displacement amount of exercise. Assuming $G(x, t)$ is an equation, which correspond to the strength of the main direction and time. If the motion changes very small, the gray value around the related point can be approximated by the first-order Taylor series.

$G(x+\Delta x, t)=G(x, t)+\frac{\partial G}{\partial x} \Delta x$

If the moving of the target at a constant speed, the gray value can be represented by an equation $\Delta x=\dot{u} \Delta t$, after a time $\Delta t$ the step

$\Delta G=G(x, t+\Delta t)-G(x, t)$

When the time step $\Delta t$ unlimited unlimited short, there are:

$\frac{\partial G}{\partial t}+\dot{u} \frac{\partial G}{\partial x}=0$

For the two-dimensional field, if the rate of movement is $\mathrm{V}$, the same first-order Taylor launched, then:

$G(\mathrm{n}+\mathrm{n} \mathrm{n})=G(\mathrm{n})+\mathrm{n} \mathrm{n} \nabla G$

So:

$\mathbf{G n} \overline{\mathbf{x}}=-\mathbf{g}$

In the least squares method for solving the average displacement $\Delta \overline{\mathrm{X}}$ :

$\mathrm{n} \overline{\mathbf{x}}=-\left(\mathbf{G}^{\top} \mathbf{G}\right)^{-1} \mathbf{G}^{\top} \mathbf{g}$

Expansion after:

$\left[\begin{array}{l}\Delta \bar{x} \\ \Delta \bar{y}\end{array}\right]=-\left[\begin{array}{ll}\sum\left(\frac{\partial G}{\partial x}\right)^{2} & \Sigma\left(\frac{\partial G}{\partial x} \frac{\partial G}{\partial y}\right) \\ \sum\left(\frac{\partial G}{\partial x} \frac{\partial G}{\partial y}\right) & \sum\left(\frac{\partial G}{\partial y}\right)^{2}\end{array}\right]^{-1}\left[\begin{array}{l}\sum \frac{\partial G}{\partial x} \Delta G \\ \sum \frac{\partial G}{\partial y} \Delta G\end{array}\right]$ 
As long as is nonsingular matrix, then this exercise is can be estimated out.These formulas are expressed in the above definition of a purely algebraic. $\|\mathbf{g}+\mathbf{G} \Delta \overline{\mathbf{x}}\|^{2}$., so now with the gray value calculate this parameter.Assuming the deformation between the two images before and after the light has not changed.F is defined as a function of the gray value of the sub-region of sample, after the displacement is $\mathrm{G}$, then the point of displacement between the two images:

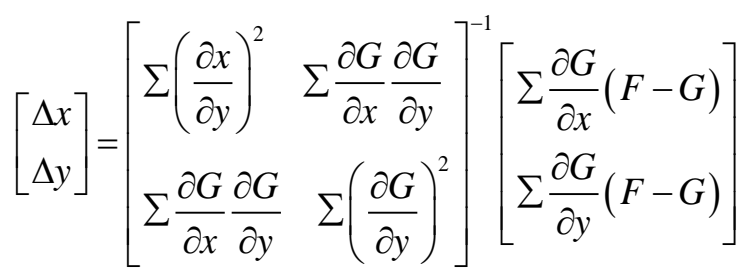

The minimax of the gray value of sum of squared differences between the two figures before and after the change in the sub-region:

$\overline{\mathrm{d}}_{o p t}=\min \sum|G(x+\bar{d})-F(x)|^{2}$

To solve this optimal displacement vectors $\overline{\mathrm{d}}_{\text {opt }}$, by extending this function then simple iterative algorithm of a first-order Taylor.

$\chi^{2}\left(\bar{d}_{x}+\Delta x, \bar{d}_{y}+\Delta y\right)=\sum\left|G(x+\bar{d})-\frac{\partial G}{\partial x} \Delta x-\frac{\partial G}{\partial y} \Delta y-F(x)\right|^{2}$

In the above formula, $d_{x}$ and $d_{y}$ are the current estimate of the average motion of the sub-regions, $\Delta x$ and $\Delta y$ are the increase in the current iteration is obtained, the $\Delta x$ and $\Delta y$ initial value is set to 0 . $\mathrm{P}$ by iteration times, improved estimate of the average exercise $\bar{d}^{p+1}=\bar{d}^{p}+\Delta$ until convergence to the optimal value of the motion vector $\bar{d}_{\text {opt }}$, then the average displacement of the two sub-region obtained.

\subsection{Related Displacement Search Method}

During the iteration, calculation is randomness. When the pixel is more, if counted individually, tend to be very large amount of computation, time-consuming and long. Before related searches, we selected the deformation in the subregion, and then assign the initial to deformation function, into their shape function, resulting in the $\left(x^{\prime}, y^{\prime}\right)$ initial value. Then selected relevant criterion function to calculate the value of $\mathrm{C}$, if $\mathrm{C}$ reaches a threshold value, you can output, if not met, then the initial value to the shape function again, this cycle. The search process is as follows Fig. (3).

In the specific search, how to reduce the number of initial values, to quickly get a peak correlation coefficient, the search method is very critical, and usually have two parts search the entire pixel and sub-pixel search. During the entire pixel search for less demanding precision, you only need to be accurate to one pixel, pixel search technology focused on the entire is able to quickly locate in a larger area.

The common integer pixel search methods:

(1) Jiabai Rui et al proposed cross search method, this method to a two-dimensional search becomes twodimensional search is undoubtedly related to the processing speed can be greatly improved, it is considered in the search

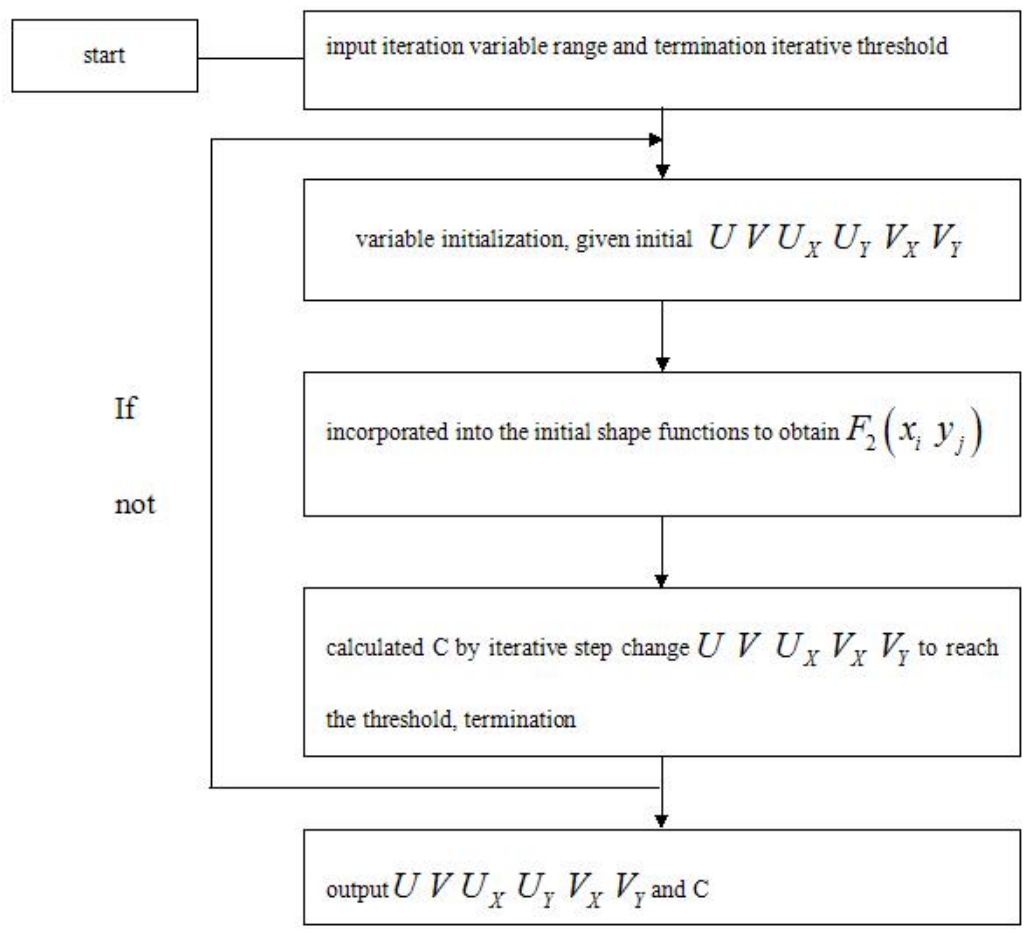

Fig. (3). Search flow chart. 
area has a single peak and main peak symmetry. Along a first direction $\mathrm{X}$ or $\mathrm{Y}$ coordinate calculations, when the maximum value obtained, and then calculate the other direction. (2) Genetic algorithms. Patent 20130173510A1, titled "METHODS AND SYSTEMS FOR USE IN REDUCING SOLUTION CONVERGENCE TIME USING GENETIC ALGORITHMS," [15] provides the method for using genetic algorithms. It is in recent years for the low quality speckle pattern and large displacement measurement arising. Solution of the problem as a population experiencing a simulated evolution, scholars have done a lot of research as Plich et al proposed genetic algorithm-based intelligent digital image correlation method. China, Pan Bing made a digital image correlation method based on differential evolution, but because these methods are difficult to preparation of a universal program, it is not practical range widely (3). Coarse - fine search method, first with a long stride for the entire search area to do the relevant calculations, find the maximum value, then the center with great value, reducing regional and step length executes correlation calculation, followed this continues until you find maximum point of the relevant coefficients. NewtonRapshon method is a common sub-pixel search method, made by Bruck and Sutton [15] in 1989, using a common numerical analysis of nonlinear equations solution, N-R iterative method to solve the extreme points of the correlation coefficient.Quasi-Newton method proposed by some scholars and it is actually similar to N-R method.There also have correlation coefficient surface (or curve) fit (or interpolation) algorithm, the correlation coefficient gradient method.

\section{APPLICATIONS}

Non-contact stress-strain tester is an instrument based on digital speckle technique. In this paper, performed axial pullout tests on CFRP embedded-style concrete specimens using non-contact stress-strain tester, analyzed the stress and strain distribution of CFRP and concrete substrate interface by SSD correlation function algorithm during the stretching.

In this experiment, the designed size of concrete specimens is $150 \mathrm{~mm} \times 150 \mathrm{~mm} \times 400 \mathrm{~mm}$, In the middle of the concrete specimen longitudinal opening a rectangular slot in which injecting epoxy resin as the bonding material, then the CFRP slats embedded into grooves, finally forming reinforced concrete beams and its bond length is $280 \mathrm{~mm}$. Before the experiment, the surface of the specimen arranged speckle and use hydraulic servo apparatus for loading 50t , showing in Fig. (4).

We shot a total of 250 specimens will face speckle pattern, from the beginning tension of the specimen to the slippage occurs between the CFRP slats and concrete bond portion, also set the speckle pattern before starting stretching as the initial state when contrasting, take more representative diagram No.120 to compare, the digital speckle as shown in Fig. (5).

When tension that is about $27.3 \mathrm{KN}$ is applied the specimen, the adhesive part of CFRP slats and epoxy resin generated debond failure. We took $4 \mathrm{KN}, 8 \mathrm{KN}, 12 \mathrm{KN}$, $16 \mathrm{KN}, 20 \mathrm{KN}$ speckle pattern when taken for analysis, the results shown in Fig. (6).
Calculation results of the analysis basically agree well with experimental failure phenomena of the specimen. From the Fig. (7) we can see, there is apparent displacement only at the top of slat that applied load, when $4 \mathrm{KN}$ received at the beginning of the tension. With the increase of tension, the displacement of the lower slat arises and concentrate on the whole slate, In addition, the area represented by the red shift downwards slowly spread. From $4 \mathrm{KN}$ to $20 \mathrm{KN}$, The overall displacement of the upper plate portion is gradually increased and some debond failure appeared on bonding interface, what is more, the bonded section between whole slat and epoxy resin generated macro debond failure. Therefore, these analytical results are basically consistent with the experimental result.

CFRP batten down every $20 \mathrm{~mm}$ from the top bonding at down to take 20 points to make displacement analysis, analyzing the longitudinal displacement of CFRP plate under different loads as shown in Fig. (5).The results show: When

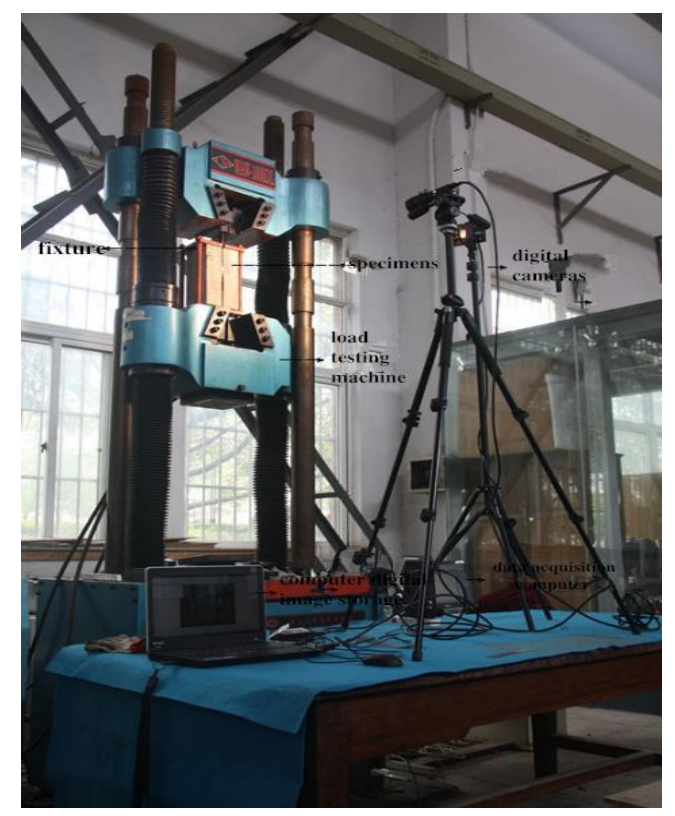

Fig. (4). Load and test device.

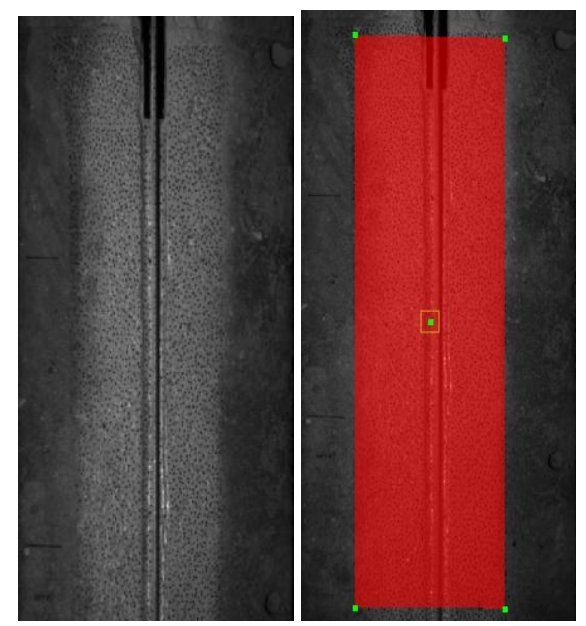

(a) Initial speckle (b) analysis region of speckle

Fig. (5). Digital speckle pattern. 

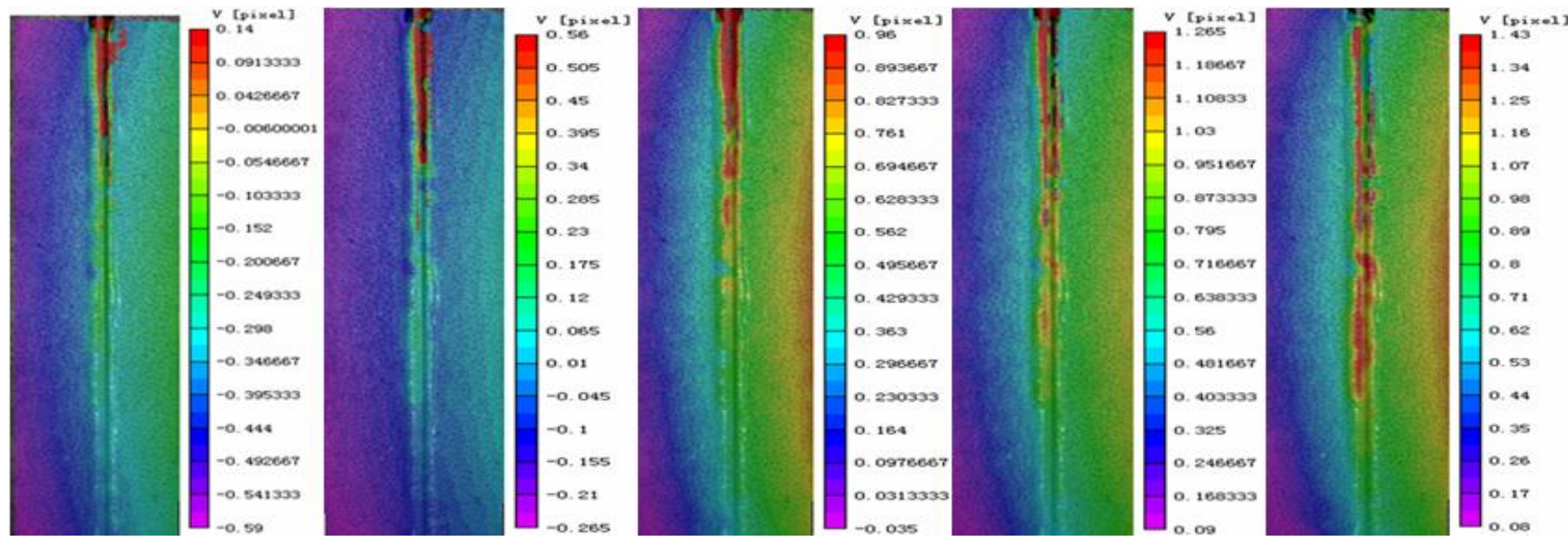

Fig.(6). Results displacement contours.

the load is small, lath displacement is small, the interfacial adhesion integrity, overall displacement is very gentle; while load gradually increasing the load displacement curve slope is growing.Especially in a place close to the loaded end distance, you can clearly see that the slope is increasing.This indicates that as the load increased, the slip at the upper end of the bond increases, when the load reaches $8 \mathrm{KN}$, interface appears slight peeling, bonding interface softening, when the load reaches $20 \mathrm{KN}$, the interface has emerged macroscopic stripping, relative slip occurred has been more obvious.

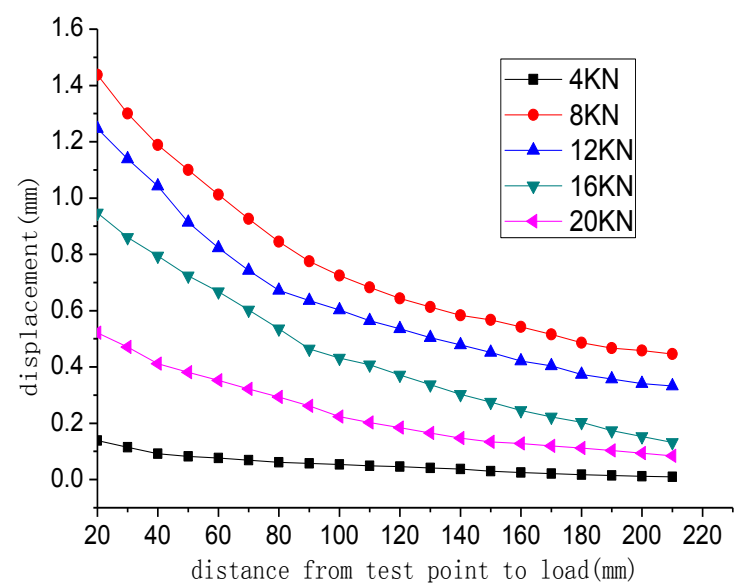

Fig.(7). CFRP batten longitudinal displacements.

\section{CONCLUSIONS}

In this paper, author calculates the basic principles of digital speckle and correlation function of SSD technology. By deriving the solving process from the gray value of the image to the specific displacement value and transforming the observed deformation of the surface into pure numerical, then understand deeply the matching principles of digital speckle technology. Launches an application experiments on the CFRP plate embedded reinforcement beam deformation and failure, which using CCD camera to collect the process of destruction caused by pulling out slats, in addition, with the SSD algorithms of vic-2d software for computational analysis .The displacement nephogram throughout the whole process of destruction has been calculated ,from which will we visually see the development of deformation, bond failure trends, and vertical displacement values of CFRP plate under different loads. Calculation results consistenting with the experimental results illustrates the accuracy and applicability of numerical speckle techniques.

Practice shows that the digital speckle technique has easy operation, non-contact without injury, the audience strain measurement, calculation accuracy, etc., can be an alternative to traditional methods of measurement, when applying to the field engineering and research activities.

\section{CURRENT \& FUTURE DEVELOPMENTS}

Digital image correlation technology has the advantages of no contact, large section testing etc., so it has been used in the fields of material forming, large deformation monitoring $[16,17]$. With the development of optical technology and the mechanical mechanism, the quality of speckle improving and 3D optical technology developing, the digital speckle correlation technology will be more rapid development.

\section{CONFLICT OF INTEREST}

The authors confirm that this article content has no conflict of interest.

\section{ACKNOWLEDGEMENTS}

This work is supported by the Chinese Natural Science Foundation (51274101, 51074076) and JiangXi Science and technology support program (20121BBG70064).

\section{REFERENCES}

[1] N. M. Gyu, and Y. S. Yeol, "Apparatus of non-contact mass measurement”, KR101363558(B1), February 17, 2014.

[2] Z. E. Gusmanovich, and N. M. Andreevich, "Device for the remote non-contact monitoring of the vital signs of a living being", $\mathrm{R}$. U. Patent 2012140602(A), March 27, 2014

[3] R. Wang, Z. Bing, D. L. Chao, and W. Xiang "Manufacturing method of high-temperature speckle", C. N. Patent 101850426(A), October 06, 2010 
[4] I. Yamaguchi, "Speckle displacement and deformation in the diffraction and image fields for small object deformation", Acta Opt. Sin., vol. 28, no. 10, pp. 1359-1376, 1981.

[5] I. Yamaguchi, "A laser-speckle strain gage", J. Phy. Eng. Sci Instrum, vol. 14, pp. 1270-1273, 1981

[6] W. H. Peters and W. F. Ranson, "Digital imaging technique in experimental Mechanics", Opt. Eng., vol. 21, no. 3, pp. 427-431, 1982.

[7] S. Zou and Y. Zeng, "Method and system for obtaining interaction information", C. N. Patent 103686256(A), March 06, 2014

[8] T. R. Lee, L. Shen, H. Zhu, J. Chu, F. Luo, and M. Liu, "Laser displacement measuring method based on digital speckle correlation method" C. N. 102967261(A), March 13, 2013.

[9] J. Gao, X. Zhou, "Digital speckle correlation method of deformation measurement", J. Exp. Mech., vol. 6, no. 4, pp. 121-127, 1991 (in Chinese).

[10] J. Gao, Digital speckle correlation method and its application in experimental mechanics, Tsinghua University: Beijing, 1989 (in Chinese)
[11] K. R. Castieman, Digital Image Processing, Tsinghua University Press; Beijing, 2000

[12] Y. An, "Electrooptical technology", Electr Ind Press, 2002, (in Chinese)

[13] D. Michael, and S. Armin, "Method for processing a digital gray value image," U. S. Patent 20080013853A1, January 17, 2008.

[14] B. Pan, K. Qian, H. Xie and A. Asundi, "Two-dimensional digital image correlation for in-plane displacement and strain measurement: A review", Meas. Sci. Tech., vol. 20, no. 6, pp. 20012004, 2009

[15] J. R. Schmid, and J. James, "Methods and systems for use in reducing solution convergence time using genetic algorithms", $\mathrm{U}$. S. Patent 20130173510A1, October 11, 2007.

[16] C. Hang, G. Yang, Y. Li, "Application of Digital image Correction Method to Test of Mechanical Properties for Weld Materials", Acta Aeron Astron Sin, 34(10): 2372-2382, 2013 (in Chinese).

[17] B. Pan, H. Xie, and Y. Xia, "Large-Deformation Measurement Based on Reliable Initial Guess in Digital Image Correlation Method", Acta. Opt. Sin., vol. 29, no. 2, pp. 400-406, 2009. (in Chinese).

(C) Liu et al.; Licensee Bentham Open.

This is an open access article licensed under the terms of the Creative Commons Attribution Non-Commercial License (http://creativecommons.org/licenses/by-nc/3.0/) which permits unrestricted, non-commercial use, distribution and reproduction in any medium, provided the work is properly cited. 